\title{
Spinocerebellar ataxia type 7
}

INSERM

\section{Source}

INSERM. (1999). Orphanet: an online rare disease and orphan drug data base.

Spinocerebellar ataxia type 7. ORPHA:94147

Spinocerebellar ataxia type 7 (SCA7), currently the only known form of autosomal dominant cerebellar ataxia type 2 (ADCA2; see this term), is a neurodeg enerative disorder characterized by progressive ataxia, motor system abnormalities, dysarthria, dysphagia and retinal degeneration leading to progressive blindness. 